\title{
Detection of Extended-Spectrum ß-lactamases Production by Escherichia coli: A Phenotypic Comparative Study
}

\section{Bilal Ahmad Tantry ${ }^{1,2}$, Abdul Hafeez Mohammed², Shaik Rahiman ${ }^{1,3}$ and Mudasar Nabi Tantry ${ }^{4}$}

${ }^{1}$ Department of Life Science, Jaipur National University, Jaipur - 302 025, India. ${ }^{2}$ Department of Pathology, Division Microbiology, College of Medicine, Aljouf University. ${ }^{3}$ Department of Pathology, Division Biochemistry, College of Medicine, Aljouf University, Sakakah, Saudi Arabia. ${ }^{4}$ Department of Clinical Biochemistry, Kashmir University, Hazratbal, Srinagar - 190 006, Jammu and Kashmir, India.

\begin{abstract}
Resistance to antibiotics is a serious concern and immediate steps are required to crub the resistance gained by microorganisms. Extended spectrum beta- lactamases (ESBLs) producing E.coli (Escherichia coli) is rapidly increasing and making antibiotics difficult to treat around the world. The screening of ESBL producers among Escherichia coli was done by method Double Disk Synergy Test (DDST), using ceftazidime and ceftriaxone disc and Combined Disc Test (CD) using ceftazidime-clavulanic acid according to Clinical and Laboratory Standard Institute (CLSI) protocol. In the DDST method, out of 60 clinical isolates of Escherichia coli, 22 were found ESBL positive isolates, and among 22 positive isolates 18 strains shows increase in zone of inhibition by both ceftazidime and ceftriaxone disc. The remaining 4 ESBL isolates, 3 of them showed zone of inhibition to ceftazidime and only 1 isolate showed inhibition by ceftriaxone disc. Similarly, in CD method apart from 22 isolates detected by DDST method it detected 1 more isolate as ESBL producers. Furthermore, the CD method detects inducible beta- lactamase in III generation of cephalosporin sensitive isolate. Our study showed 22 isolates were found by DDST method and 23 isolates by CD method. This study results showed the prevalence of ESBL in $E$. coli were $36.66 \%$ by DDST and $38.33 \%$ by CD tests and we conclude that combined disk test (CD) is better than DDST for detection of ESBL.
\end{abstract}

Keywords: Extended-spectrum $\beta$-lactamase, Escherichia coli, Antibiotics, India.

*Correspondence: bilaltantry@gmail.com; +919797915758.

(Received: 15 November 2018; accepted: 20 December 2018)

Citation: Bilal Ahmad Tantry, Abdul Hafeez Mohammed, Shaik Rahiman and Mudasar Nabi Tantry, Detection of Extended-Spectrum ß-lactamases Production by Escherichia coli: A Phenotypic Comparative Study, J Pure Appl Microbiol., 2018; 12(4):2245-2252. http://dx.doi.org/10.22207/JPAM.12.4.64

(c) The Author(s) 2018. Open Access. This article is distributed under the terms of the Creative Commons Attribution 4.0 International License which permits unrestricted use, sharing, distribution, and reproduction in any medium, provided you give appropriate credit to the original author(s) and the source, provide a link to the Creative Commons license, and indicate if changes were made. 


\section{INTRODUCTION}

The beta lactam antibiotics are hydrolyzed by Extended-spectrum $\beta$-lactamases (ESBL) producing microorganism (Klebsiella species, Acinetobacter, Pseudomonas aeruginosa, Citrobacter, Proteus, Enterobacter and Escherichia coli ) and is a major challenge in infectious diseases (Aminzadeh Z et al., 2008; Khanfar et al.,2009). Antibiotic resistant strains of Escherichia coli produce an enzyme known as ESBL and such strains are resistant to other class of antibiotic (Jacoby GA et al., 2005; Livermore DM et al., 2007). This makes the pathogen hard to treat. ESBL producing strains cause a wide range of infections from septicemia to urinary tract infection (Thakur $S$ et al., 2013). The main target of ESBL producing pathogens are elderly people and those who have been recently admitted in the hospital and are taking antibiotics. The ESBLs are typically plasmid mediated enzymes that hydrolyze penicillin, thirdgeneration azetreonam and cephalosporin. They are not active against cefotetan, cefoxitin and cephamycins but are susceptible to clavulanic acid. Other, Antibiotics classes like, fluroquinolones and aminoglycosides the ESBL are resistant (Egea $P$ et al., 2011; KO ks et al., 2007; Yadav et al., 2015). Plasmids play an important role in resistance to cephamycins involving amp $\mathrm{C}$ gene. The oxyimino group addition to the C-7-amino position of second and third generation of cephalosporin results in the broad spectrum cover to Enterobacteriaceae. The attachment of beta lactamase to the antibiotic is prevented by oxyimino group (Kangh et al., 2005; Sachin et al., 2011). Furthermore, uncontrolled use of aztreonam and cephalosporin (Third generation cephalosporin) results in the mutation in the enzymes and which in turn cause the emerge of ESBL producing pathogens (Grover et al., 2006; Sharma et al., 2013; Kumar et al., 2014). The double disk test method is primarily used to detect the ESBL producing microorganism. The aim of this study is to find the ESBL producing $E$. coli from non ESBL producing strains.

\section{MATERIALS AND METHODS}

The clinical isolates of $E$. coli strains were obtained from specimens (urine, blood, wound, pus, and semen) from patients admitted in different wards of Fortis Hospital, Noida, India between February 2016 to May 2017 following standard procedures CLSI. A total of 60 samples of $E$. coli were collected of all age groups. After receiving the clinical isolates in the laboratory they were immediately cultured on Mac Conkey agar for further study. Standard protocol was followed for the identification of E.coli (Collee et al.,1996; Bailey and Scott's 2007). Permission for work was approved by the Ethical committee of the institute and consent from patients.

\section{Extended-spectrum $\beta$-lactamaseScreening of the isolates}

An inoculum of 0.5 McFarland dilutions of all test strains, negative control and positive control were prepared in $3 \mathrm{ml}$ of normal saline. Streak a lawn of all dilution of all test strains, negative control and positive control to a Mueller Hinton agar plate and allow to dry 3-5 minutes. After drying, an amoxicillin-clavulanic acid (20/10 $\mu \mathrm{g})$ disc was placed at the center of the plate. A disc of ceftazidime $(30 \mu \mathrm{g})$ and ceftriaxone $(30 \mu \mathrm{g})$ were placed one on either side of amoxicillin-clavulanic acid $(20 / 10 \mu \mathrm{g})$ disc at a distance of $15 \mathrm{~mm}$ from center to center. All plates were incubated at $35^{\circ} \mathrm{C} \pm 2{ }^{\circ} \mathrm{C}$ in incubator for $16-24$ hours. E.coli (ATCC 25922) and K.pneumoniae (ATCC 700603) producing beta-lactamase were used as Negative control and positive control. After the incubation of $24 \mathrm{hr}$ the culture plate was studied for zone of inhibition in an area between ceftriaxone and amoxicillin clavulanic acid in comparison with the zone of inhibition on the far side of these drugs was taken as a positive.

\section{Double disc synergy test (DDST)}

Muller Hinton Agar culture plate was used as standard disk diffusion assay. The ceftriaxone of 30 microgram and cefazidime of 30 microgram (15 mm apart), including a disk of augmentin 20 +10 micrograms) disks were used for screening of ESBL producing microorganism (Wayne, PA. 2013). The zone of inhibition of either ceftriaxone or cefazidime towards amoxicillin and clavulanic acid is considered as ESBL producing pathogen and is known as confirmatory phenotypic test.

\section{Combined Disc Test (CD)}

The ESBL producing group I (class A) microorganism are inhibited by clavulanic acid and for such type of stains third generation of cephalosporins along with clavulanic acid can be used as confirmatory test (Kang HY et al., 2005). If the zone of inhibition restricts to third generation 
of cephalosporin only, than it can be considered as confirmatory test for ESBL. An inoculum of 0.5 McFarland dilutions of all test strains, negative control and positive control were prepared in $3 \mathrm{ml}$ of normal saline with the help of 0.5 McFarland standards. Streak a lawn of all dilution of all test strains, negative control and positive control to a Mueller Hinton agar plate and allow to dry 5 minutes, than aspetically a disk of ceftazidime $(30 \mu \mathrm{g})$-clavulanic acid $(10 \mu \mathrm{g})$ and ceftazidime $(30 \mu \mathrm{g})$ were placed at a $30 \mathrm{~mm}$ of distance. All plates were incubated at $35^{\circ} \mathrm{C}$ in incubator for 24 hours. Non-ESBL producing organism E.coli and an ESBL-producing organism $K$. pneumoniae were used as negative and positive controls respectively. After $18 \mathrm{hr}$ of incubation the culture plate is examined for the zone of inhibition. The $5 \mathrm{~mm}$ zone of inhibition of (Ceftazidime-Clavulanic acid) is greater than that of ceftazidime than it will be taken as positive for ESBL production.

\section{RESULTS}

\section{Double Disk Synergy Test (DDST)}

A total of 60 non duplicate isolates of $E$. coli were included in the study. Out of 60 strain, $46(76.66 \%)$ were isolated from urine, $4(6.66 \%)$ from wound, 4 (6.66\%) from pus, 2 (33.33\%) from semen and 4 (6.66\%) from blood (Figure 1). All 60 non duplicate isolates of $E$. coli isolates were tested by DDST, detected 22 (36.66\%) strains as ESBL producers (Figure 2), among which 15(25\%) isolates from urine, 2 (3.33\%) from pus, 2 (3.33\%) from wound, 2 (3.33\%) from blood and 1 (1.66\%) from semen (Figure 3 ). Out of 22 ESBL positive isolates, 18 isolates produced increase zone of inhibition by both ceftazidime and ceftriaxone disc (Figure 4). While in remaining 4 isolates, 3 isolates produced increase zone of inhibition by only ceftazidime and 1 isolates by ceftriaxone disc.

\section{Combined Disc Test (CD)}

All 60 non duplicate isolates of $E$. coli isolates were again tested by Combined Disc Test, detected 23(38.33\%) strains as ESBL producers, among which 16 (26.66\%) isolates from urine, 2 (3.33\%) from pus, 2 (3.33\%) from wound, 2 (3.33\%) from blood and 1 (1.66\%) from semen (Figure 3). The isolates exhibited a significant zone size enhancement with the combination disk (ceftazidime -clavulanic acid) when compared to the plain antibiotic (ceftazidime) discs (Figure 5).
The CD detected the same 22 strains of DDST as well as additional 1 strain as ESBLs producers. The CD test also detects inducible B- lactamase in $3^{\text {rd }}$ generation cephalosporine sensitive isolates. Only one isolate is sensitive to ceftazidime but it is detected as ESBL producer.

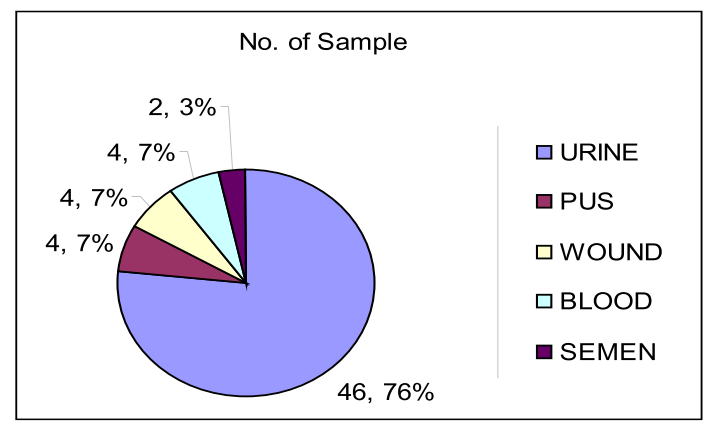

Fig. 1. Distribution of sample collection.

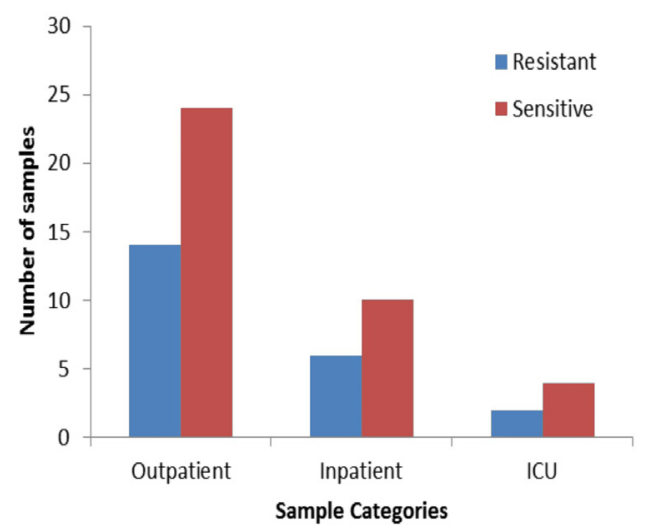

Fig. 2. Distribution of antibiotic resistance pattern of Escherichia coli isolated from patients

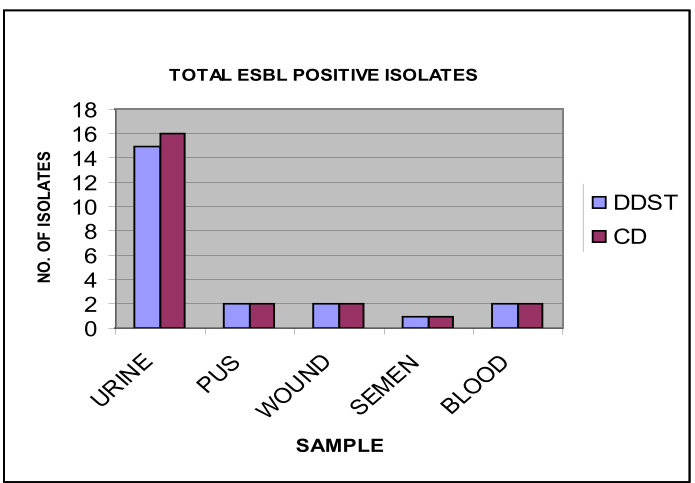

Fig. 3. Extended spectrum beta- lactamases producing isolates from various samples using phenotypic methods Double Disk Synergy Test( DDST ) and Combined Disc Test (CD) method. 
Among 23(42\%) metallo beta lactamase (MBLs) producing isolates, $16(30 \%)$ isolates $E$. coli exhibited zone of inhibition difference between combination disk (ceftazidime -clavulanic acid) and ceftazidime disc alone were 6 to $10 \mathrm{~mm}$. Remaining 7 (12\%) isolates exhibited e" $10 \mathrm{~mm}$ difference zone. Remaining $47 \mathrm{E}$. coli isolates had a smaller difference (less than $5 \mathrm{~mm}$ ) in zone of inhibition around the combined disk than around the ceftazidime disc alone and thus they were considered to be non ESBL producer.

Comparison between double disk synergy test and combined disc test

The possible presence of ESBL-producing strains was tested among $60 \mathrm{E}$. coli strain by Double Disk Synergy Test (DDST) and Combined

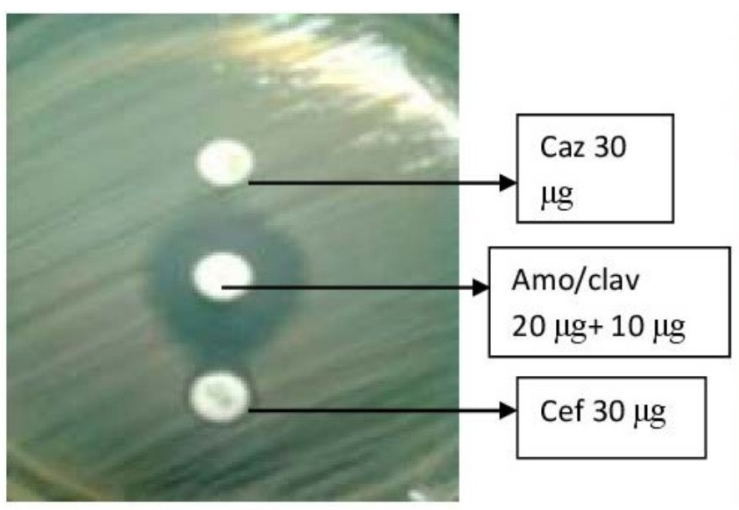

A) Positive DDST Test

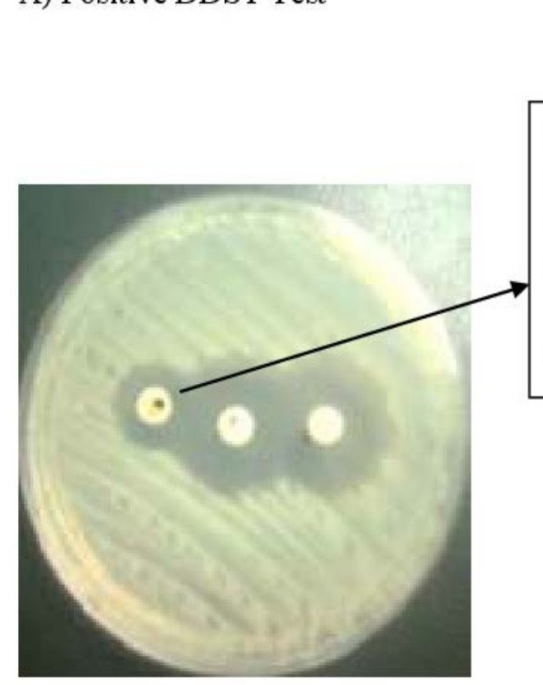

C) Positive DDST Test
Disc Test (CD) (Figure 3). Above two methods DDST and CD detected ESBLs producer isolates were $22(36.66 \%)$ and $23(38.33 \%)$ respectively (Table 1). The CD detected the same 22 strains of DDST as well as additional 1 strain as ESBL producers. When procedures of DDST were repeated for above 1 (firstly negative) strain these become positive for ESBL in repeated DDST test. The phenotypic methods CD and DDST are most reliable for ESBL detection in E coli. However, the DDST has a disadvantage as distance varies with the strains. Our study confirms that the CD is far more accurate than DDST for ESBL producing bacteria. This method can be used a key to monitor ESBL emergence.

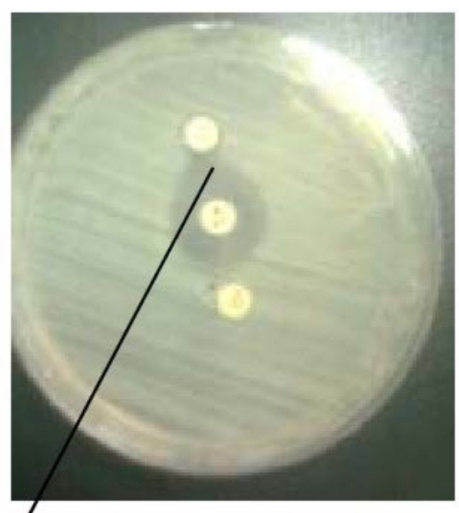

B) Positive DDST Test

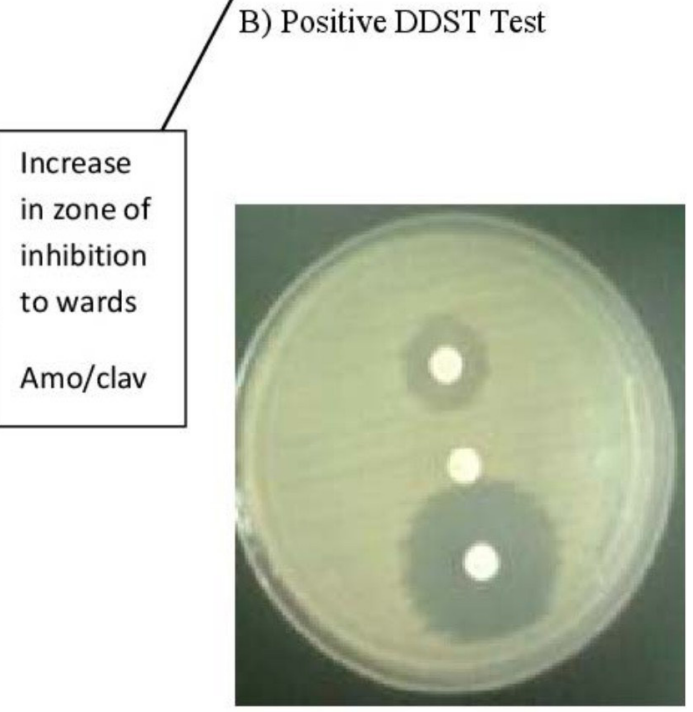

D) Negative DDST Test

Fig. 4. Extended-spectrum ß-Lactamase (ESBL) Positive Isolates by Double Disc Synergy Test (CD) 


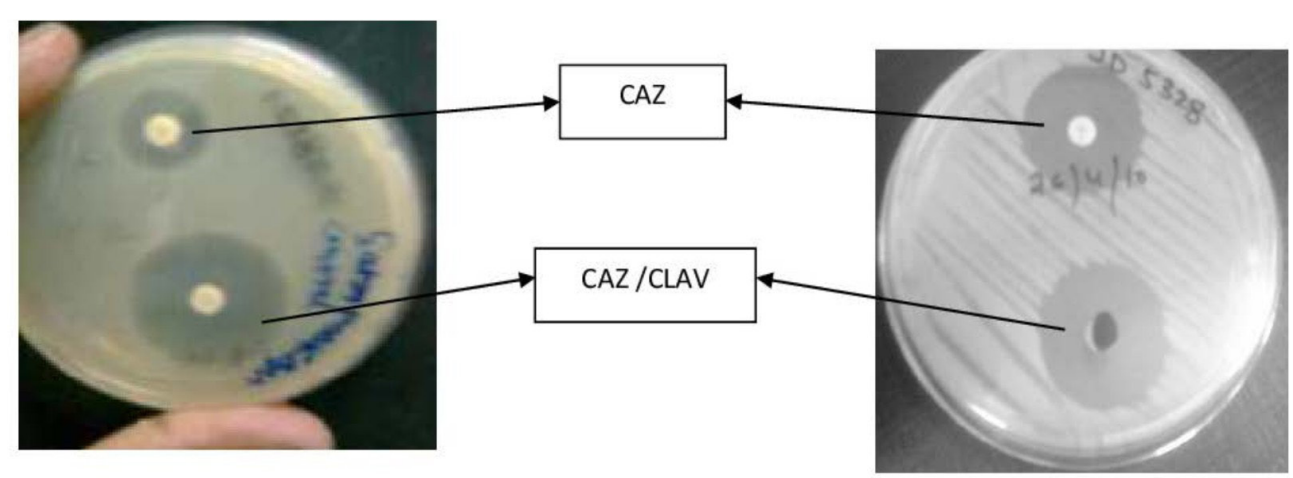

A) Positive Control K. pneumoniae (ATCC 700603)

B) NegativE control.

E. coli (ATCC 25922)

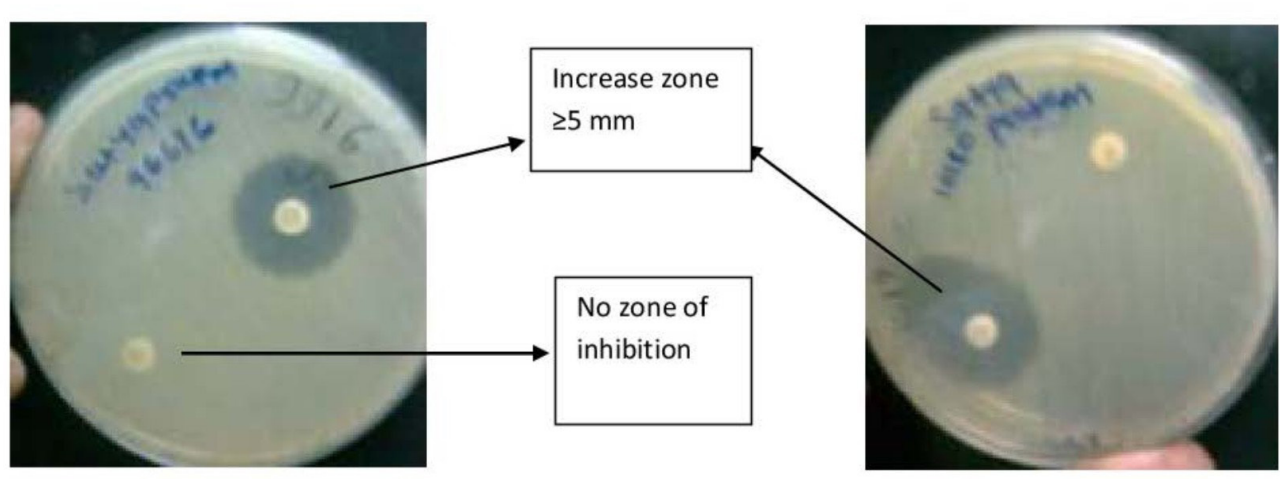

C) ESBL Positive Isolates

D) ESBL Positive Isolates

Fig. 5. Extended-spectrum ß-Lactamase (ESBL) Positive Isolates by Combined Disc Test (CD)

Table 1. The number of ESBL producing E. coli in various samples by phenotypic method.

\begin{tabular}{lcc}
\hline Sample & DDST & CD \\
\hline Urine & 15 & 16 \\
Pus & 2 & 2 \\
Wound & 2 & 2 \\
Semen & 1 & 1 \\
Blood & 2 & 2 \\
\hline
\end{tabular}

\section{DISCUSSION}

In the developing nations the emergence of ESBL producing pathogens is high and causing serious issues worldwide (KMendelsonG et al.,2005; Kumar et al., 2006; Bazzaz et al., 2009; ronenberg et al., 2013). In United States the E. coli and Klebsiella pneumonia are associated with ESBL production, hence; efficient methods are required to rapid detection of the ESBL producing pathogens of Enterobacteriaceae family (Kumar et al., 2006). Conventional susceptibility testing methods, on their own, fail to offer reliable susceptibility results for $\beta$-lactam antibiotics when testing those species that harbor ESBLs. 30-90 \% of ESBL producers are found in various hospitals (Yadav KK et al., 2015). Our study shows the occurrence of ESBL was $36.66 \%$ in E. coli by DDST method and $38.33 \%$ by CD method, we are in agreement with DDST method which shows $95.65 \%$ as compared to CD test. The antibiotic resistance is a major issue to public health and patient care. The $E$. coli is highly resistant to penicillin and less 
resistant to imepenem (ZhanelGG et al., 2008). ESBL producing pathogens created a significant issues as these microbes are resistant to wide range of antimicrobials (third generations of cephalosporins) and also have plasmid borne resistance to carbapenem(Lautenbach et al., 2001; Villa et al., 2000). Multidrug resistance has complicated the antibiotic choice (Rath $\mathrm{S}$ et al., 2014). Our study shows there is increase in ESBL producing pathogens with time and there is need of control measures to be taken to stop misuse of antibiotics. Amakacin remain a drug of choice to treat the diseases caused by ESBL producing pathogens. This class of drug can be used to prevent the spread of ESBL resistance frequency. Molecular work is required to know more about the genes in the ESBL producing pathogens

Poor sensitivity in DDST mainly due to the lack of optimum disc space results in the impaired ESBLs inhibition by clavulanate leads to the inaccurate identification of chromosomal cephalosporinases producing ESBLs strain in the Muller-Hinton agar medium. Earlier studies reports various alternative methods to overcome this disadvantage of DDST such as reduction of disk space from $20 \mathrm{~mm}$ to $30 \mathrm{~mm}$ to obtain more accurate results (Thomson KS et al.,1992) whereas, in our study we placed the ceftazidime and ceftriaxone disks at a distance of $15 \mathrm{~mm}$ from AMC disk to obtain more sensitive results as reported by Coudron PE et al., 1997).

The primary initial screen test for ESBL positive E.coli isolates is CLSI method in many hospitable based microbiology laboratories. The delayed results from this CLSI method were may adversely affect the patient treatment; hence, there is a need to develop a reliable, sensitive, and rapid ESBIL detection method. In this regards, in this study we tested an accelerated hybrid protocol to overcome the cons of the CLSI method. In our study we screen the ESBL strains by incorporated the combined disk test in the standard KB (KirbyBauer) disk diffusion antimicrobial susceptibility test to obtain the more accurate and rapid sensitive results. This new practiced hybrid protocol allowed to detecting the ESBL producing strain same day along with the first line antimicrobial susceptibility test and aided to improve the patient care.

In our study, we found that the combined disk test is reliable, convenient, cheap and easy to perform than double disk synergy test. It also reduces the time of reporting and antimicrobial therapy. By placing a disc of ceftazideme / clavulanic acid as extra disc for ESBL detection in E. coli routine susceptibility on MHA plate, the comparison between zone of inhibition difference ceftazideme and ceftadideme / clavulanic acid gives early detection of ESBL producing isolates. The sensitivity and specificity of combined disc test is higher than that of the DDST as described above. Thus combined disk test (CD) can be easily used in routine laboratory of developing countries including India.

\section{CONCLUSION}

Our study validates a simple and highly sensitive phenotypic method for the detection of ESBL production in E. coli. Based on the findings of our study we conclude that CD is better than DDST for detection of ESBL. As, the CD test was able to detect more than one strain whereas, DDST test limited to single strain only. Furthermore, CD test has high specificity and sensitivity than DDST. In our study the prevalence of ESBL in E. coli were $36.66 \%$ by DDST and $38.33 \%$ by CD tests.

\section{ACKNOWLEDGMENTS}

I would like to express my special thanks of gratitude to $\mathrm{Dr}$ Avineesh Kumar (Asst Professor, Microbiology), who gave me the golden opportunity to do this wonderful project.

\section{REFERENCES}

1. Aminzadeh Z, Kashi MS, Shaabani M. Bacteriuria by extended-spectrum beta-lactamaseproducing Escherichia coli and Klebsiella pneumoniae isolates in a governmental hospital in south of Tehran, Iran. 2008.

2. Khanfar HS, Bindayna KM, Senok AC, Botta GA. Extended spectrum beta-lactamases (ESBL) in Escherichia coli and Klebsiella pneumoniae: trends in the hospital and community settings. The Journal of Infection in Developing Countries. 2009; 3(04):295-9.

3. Jacoby GA, Munoz-Price LS. The new $\beta$-lactamases. New England Journal of Medicine. 2005; 352(4):380-91.

4. Livermore DM, Canton R, Gniadkowski $M$, Nordmann P, Rossolini GM, Arlet G, et al. CTX-M: changing the face of ESBLs in Europe. Journal of Antimicrobial Chemotherapy. 2007; 59(2):16574. 
5. Thakur S, Pokhrel N, Sharma M. Prevalence of multidrug resistant Enterobacteriaceae and extended spectrum $\beta$-lactamase producing Escherichia coli in urinary tract infection. Res $J$ Pharm Biol Chem Sci. 2013; 4(2):1615.

6. $\quad$ Egea P, Lopez-Cerero L, Navarro M, RodriguezBano J, Pascual A. Assessment of the presence of extended-spectrum beta-lactamase-producing Escherichia coli in eggshells and ready-toeat products. European journal of clinical microbiology \& infectious diseases. 2011; 30(9):1045.

7. Ko KS, Suh JY, Peck KR, Lee MY, Oh WS, Kwon $K T$, et al. In vitro activity of fosfomycin against ciprofloxacin-resistant or extended-spectrum $\beta$-lactamase-producing Escherichia coli isolated from urine and blood. Diagnostic microbiology and infectious disease. 2007; 58(1):111-5.

8. Yadav KK, Adhikari N, Khadka R, Pant AD, Shah B. Multidrug resistant Enterobacteriaceae and extended spectrum $\beta$-lactamase producing Escherichia coli: a cross-sectional study in National Kidney Center, Nepal. Antimicrobial resistance and infection control. 2015; 4(1):42.

9. Kang HY, Jeong YS, Oh JY, Tae SH, Choi CH, Moon DC, et al. Characterization of antimicrobial resistance and class 1 integrons found in Escherichia coli isolates from humans and animals in Korea. Journal of Antimicrobial Chemotherapy. 2005; 55(5):639-44.

10. Schink A-K, Kadlec K, Schwarz S. Analysis of blaCTX-M-carrying plasmids from Escherichia coli isolates collected in the BfT-GermVet study. Applied and environmental microbiology. 2011; 77(20):7142-6.

11. Grover S, Sharma M, Chattopadhya D, Kapoor $\mathrm{H}$, Pasha S, Singh G. Phenotypic and genotypic detection of ESBL mediated cephalosporin resistance in Klebsiella pneumoniae: emergence of high resistance against cefepime, the fourth generation cephalosporin. Journal of Infection. 2006; 53(4):279-88.

12. Kumar D, Singh AK, Ali MR, Chander $\mathrm{Y}$. Antimicrobial susceptibility profile of extended spectrum $\beta$-lactamase (ESBL) producing Escherichia coli from various clinical samples. Infectious Diseases: Research and Treatment. 2014; 7:IDRT. S13820.

13. Sharma M, Pathak S, Srivastava P. Prevalence and antibiogram of Extended Spectrum b-Lactamase (ESBL) producing Gram negative bacilli and further molecular characterization of ESBL producing Escherichia coli and Klebsiella spp. Journal of clinical and diagnostic research: JCDR. 2013; 7(10):2173.
14. Collee J, Marr W. Culture of bacteria. Mackie and McCartney practical medical microbiology. 1996; 14:113-29.

15. Forbes B, Sahm D, Weissfeld A. Overview of bacterial identification methods and strategies. Bailey and Scott's diagnostic microbiology12th Edn, eds Allen A, Wilson L, Wurm E, editors(Philadelphia, PA: Mosby Elsevier. 2007; 216-47.

16. CLSI. Performance standards for antimicrobial susceptibility testing. Clinical and Laboratory Standards Institute, Wayne, PA. 2013(CLSI document ):M100-S20.

17. Bazzaz B, Naderinasab M, Mohamadpoor A, Farshadzadeh Z, Ahmadi S, Yousefi F. The prevalence of extended-spectrum beta-lactamase-producing Escherichia coli and Klebsiella pneumoniae among clinical isolates from a general hospital in Iran. Acta microbiologica et immunologica Hungarica. 2009; 56(1):89-99.

18. Kronenberg A, Hilty M, Endimiani A, Mühlemann K. Temporal trends of extended-spectrum cephalosporin-resistant Escherichia coli and Klebsiella pneumoniae isolates in in-and outpatients in Switzerland, 2004 to 2011. Euro surveillance. 2013; 18(21):20484.

19. Kumar M, Lakshmi V, Rajagopalan R. Occurrence of extended spectrum beta-lactamases among Enterobacteriaceae spp. isolated at a tertiary care institute. Indian journal of medical microbiology. 2006; 24(3):208.

20. Mendelson G, Hait V, Ben-Israel J, Gronich D, Granot E, Raz R. Prevalence and risk factors of extended-spectrum beta-lactamase-producing Escherichia coli and Klebsiella pneumoniae in an Israeli long-term care facility. European journal of clinical microbiology and infectious diseases. 2005; 24(1):17-22.

21. Zhanel GG, DeCorby M, Laing N, Weshnoweski B, Vashisht R, Tailor F, et al. Antimicrobial-resistant pathogens in intensive care units in Canada: results of the Canadian National Intensive Care Unit (CAN-ICU) study, 2005-2006. Antimicrobial Agents and Chemotherapy. 2008; 52(4):1430-7.

22. Lautenbach E, Strom BL, Bilker WB, Patel JB, Edelstein PH, Fishman NO. Epidemiological investigation of fluoroquinolone resistance in infections due to extended-spectrum $\beta$-lactamase-producing Escherichia coli and Klebsiella pneumoniae. Clinical Infectious Diseases. 2001; 33(8):1288-94.

23. Villa L, Pezzella C, Tosini F, Visca P, Petrucca A, Carattoli A. Multiple-antibiotic resistance mediated by structurally related IncL/M plasmids 
carrying an extended-spectrum $\beta$-lactamase gene and a class 1 integron. Antimicrobial agents and chemotherapy. 2000; 44(10):2911-4.

24. Rath S, Dubey D, Sahu MC, Debata NK, Padhy RN. Surveillance of ESBL producing multidrug resistant Escherichia coli in a teaching hospital in India. Asian Pacific journal of tropical disease. 2014; 4(2):140-9.

25. Thomson KS, Sanders CC. Detection of extendedspectrum beta-lactamases in members of the family Enterobacteriaceae: comparison of the double-disk and three-dimensional tests. Antimicrobial Agents and Chemotherapy. 1992; 36(9):1877-82.

26. Coudron PE, Moland ES, Sanders CC. Occurrence and detection of extended-spectrum betalactamases in members of the family Enterobacteriaceae at a veterans medical center: seek and you may find. Journal of clinical Microbiology. 1997; 35(10):2593-7. 